\title{
Reduction in quality of skeletal muscle may have an unfavorable impact on functional prognosis in patients with metastatic spine tumors receiving non-surgical treatment. \\ M Kitagawa ${ }^{12}$, T Tsuji ${ }^{3}$, T Okayama ${ }^{1}$, Y Masuda ${ }^{1}$, T Ishii ${ }^{1}$, N Mitsuhashi ${ }^{1}$, Y Yonenaga ${ }^{1}$, N Konishi ${ }^{2}$, T Naito ${ }^{4}$, H Katagiri ${ }^{5}$, K Mori ${ }^{6}$, A Tanuma ${ }^{1}$, M Liu ${ }^{3}$ \\ 1. Division of Rehabilitation Medicine, Shizuoka Cancer Center, Shizuoka, Japan \\ 2. Department of Rehabilitation Medicine, Keio University Graduate School of Medicine, Tokyo, Japan \\ 3. Department of Rehabilitation Medicine, Keio University School of Medicine, Tokyo, Japan \\ 4. Division of Thoracic Oncology, Shizuoka Cancer Center, Shizuoka, Japan \\ 5. Division of Orthopaedic Oncology, Shizuoka Cancer Center, Shizuoka, Japan \\ 6. Division of Clinical Research Center, Shizuoka Cancer Center, Shizuoka, Japan
}

\section{BACKGROUND}

Patients with metastatic spine tumors may have an increased risk for losing skeletal muscle and decreasing physical function due to bed rest and restrictions in movements. The purpose of this study was to clarify the relationship between changes in skeletal muscle and functional prognosis in those patients.

\section{METHODS}

[Design] Retrospective study

[Period] 2014/04/01-2017/04/31

[Place] Shizuoka Cancer Center

[Inclusion criteria]

Inpatients with metastatic spine tumors (either cervical, thoracic, or lumbar vertebrae) who received physiotherapy and radiotherapy performed at doses greater than 8Gy.

\section{[Objective variable]}

() Functional prognosis: walking ability (poor / good) at T1

«criteria for poor walking》

- non-ambulatory

- need more assistance than contact

- spend the whole day in bed except during toileting and meals

[Explanatory variables]

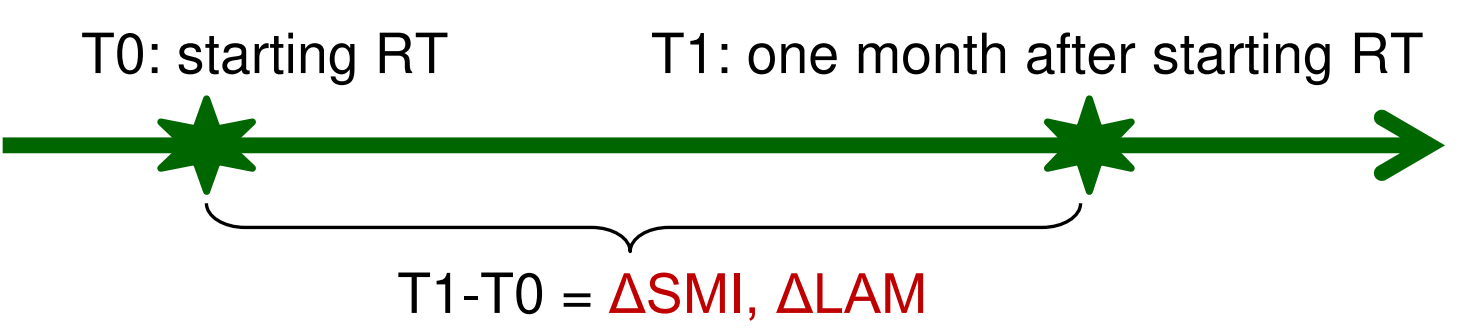

(0) Quantity of muscle (Skeletal Muscle Index; SMI)

$\mathrm{SMI}=$ Cross-sectional area on L3 slice CT/height ${ }^{2}$ at whole lumbar, psoas major, and paraspinal muscles.

() Quality of muscle (Low attenuation muscle area; LAM) $\mathrm{LAM}=$ ratio of decreased CT value $(-29$ to $+29 \mathrm{HU})$ to crosssectional skeletal muscle $(-29$ to $+150 \mathrm{HU})$ at Whole lumbar, psoas major, and paraspinal muscles.
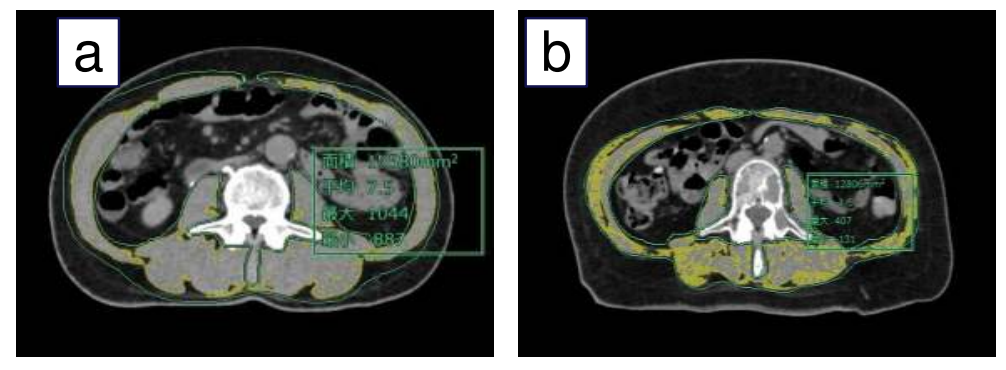

Figure1. Low Attenuation Muscle Area; LAM

a. high quality muscle (LAM $17.7 \%$ )

b. low quality muscle (LAM $46.7 \%$ )

\section{[Statistical analyses]}

\footnotetext{
1) The change of SMI and LAM between T0 and T1

$\Rightarrow$ Wilcoxon's signed-rank sum test.
}

2) The effect of $\Delta S M I$ and $\triangle \mathrm{LAM}$ on functional prognosis, or walking ability at $\mathrm{T} 1 . \Rightarrow$ Single logistic regression analysis.

\section{RESULTS}

[Patients] Date from a total of 29 patients were collected.

Median age: 66 years $(30-86)$

Primary tumors: Lung 48\%, Kidney $10 \%$, Double cancer $7 \%$, et al.

Functional prognosis: poor $17.2 \%$, good $82.8 \%$

\section{[Analysis Result]}

SMI decreased significantly in all skeletal muscles between T0-T1.

LAM tended to increase, but there were no significant difference.

$\mathrm{cm}^{2} / \mathrm{m}^{2}$

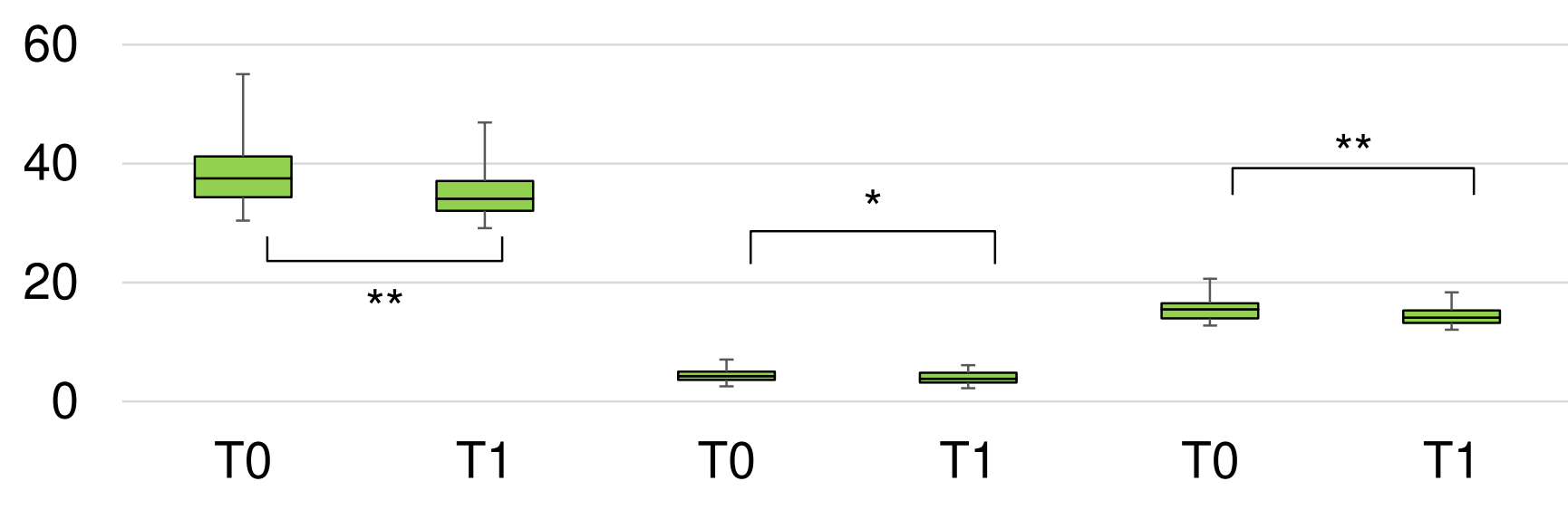

Whole lumbar muscles psoas major muscle paraspinal muscles

Figure 2 Transition of SMI $\left(p<0.01 * *, p<0.05^{*}\right)$

$80 \%$

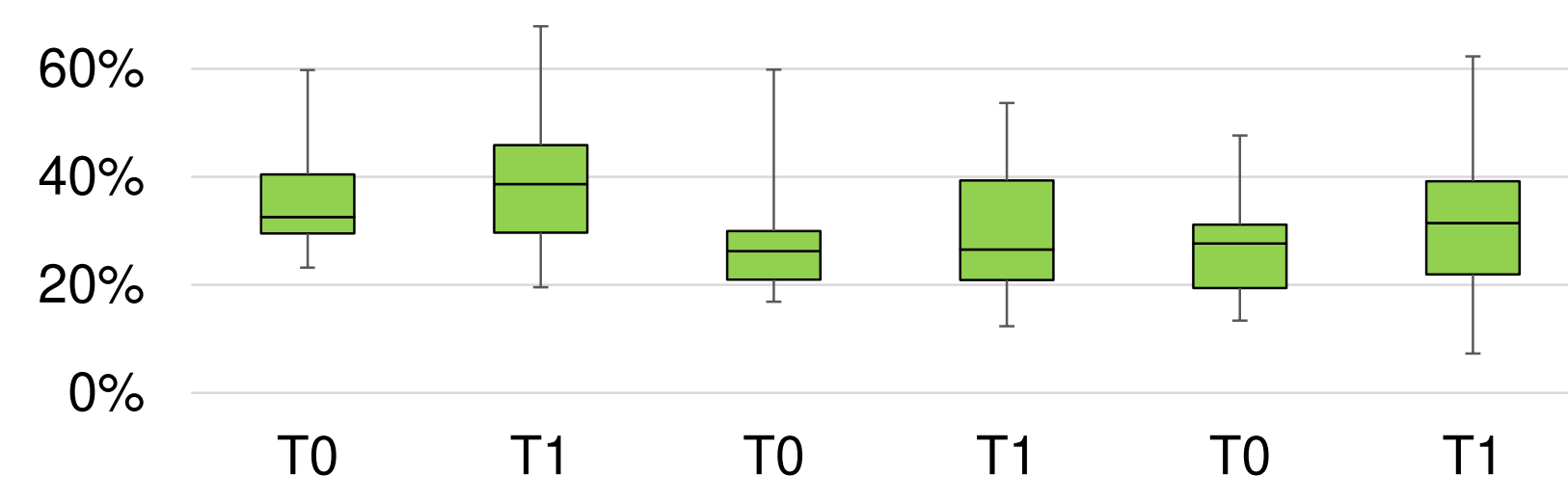

Whole lumbar muscles psoas major muscle paraspinal muscles

Figure 3 Transition of LAM

Variables significantly related to functional prognosis were $\triangle \mathrm{LAM}$ of the whole lumbar muscle, psoas major muscle, and paraspinal muscle.

Table1 The effect of $\Delta S M I$ and $\Delta L A M$ on functional prognosis

\begin{tabular}{clrrrr} 
variables, median & \multicolumn{2}{c}{$\begin{array}{c}\text { functional } \\
\text { prognosis }\end{array}$} & $\begin{array}{l}\text { odds } \\
\text { ratio }\end{array}$ & p value \\
& poor & good & & \\
\hline \multirow{2}{*}{$\Delta$ SMI } & phole lumbar muscles & -2.1 & -3.7 & 0.99 & 0.97 \\
& psoas major muscle & -0.4 & -0.1 & 0.65 & 0.60 \\
& paraspinal muscles & -1.5 & -1.1 & 1.20 & 0.57 \\
\hline \multirow{2}{*}{$\Delta$ LAM } & whole lumbar muscles & +17.3 & 0.0 & 23.87 & $<0.01$ \\
& psoas major muscle & +13.2 & -1.0 & 5.22 & $<0.01$ \\
& paraspinal muscles & +12.2 & +1.9 & 5.04 & $<0.01$ \\
\hline
\end{tabular}

\section{CONCLUSION}

After radiation therapy, quantity of skeletal muscle decreased significantly. It was also revealed that patients with reduced quality of skeletal muscle had poorer functional prognosis one month after starting radiotherapy. It could be important to maintain the quantity and quality of skeletal muscle. 